\title{
Evidence for a Solid-Electrolyte Inductive Effect in the Superionic Conductor $\mathrm{Li}_{10} \mathrm{Ge}_{1-\mathrm{x}} \mathrm{Sn}_{\mathrm{x}} \mathrm{P}_{2} \mathrm{~S}_{12}$
}

Sean P. Culver ${ }^{\mathrm{a}, \mathrm{b}, \$}$, Alexander G. Squires ${ }^{\mathrm{c}, \mathrm{d}, \$}$, Nicolò Minafra ${ }^{\mathrm{e}}$, Callum W. F. Armstrong ${ }^{\mathrm{c}}$, Thorben Krauskopfa, ${ }^{\mathrm{a},}$, Felix Böchera, ${ }^{\mathrm{a}, \mathrm{b}}$ Cheng Li ${ }^{\mathrm{f}}$, Benjamin J. Morgan*c,d,

Wolfgang G. Zeier*,e

anstitute of Physical Chemistry, Justus-Liebig-University Giessen, Heinrich-Buff-Ring 17, D35392 Giessen, Germany.

${ }^{b}$ Center for Materials Research (LaMa), Justus-Liebig-University Giessen, Heinrich-Buff-Ring 16, D-35392 Giessen, Germany.

${ }^{c}$ Department of Chemistry, University of Bath, Claverton Down, UK

${ }^{d}$ The Faraday Institution, Didcot OX11 ORA, U.K.

${ }^{e}$ Institute of Inorganic and Analytical Chemistry, University of Münster, Correnstrasse 30, 48149 Münster, Germany

fJülich Centre for Neutron Science JCNS, Forschungszentrum Jülich GmbH, Outstation at SNS, 1 Bethel Valley Road, Oak Ridge, Tennessee 37831-6473, United States.

*B.J.Morgan@bath.ac.uk; wzeier@uni-muenster.de

${ }^{\$}$ These authors contributed equally to the manuscript 


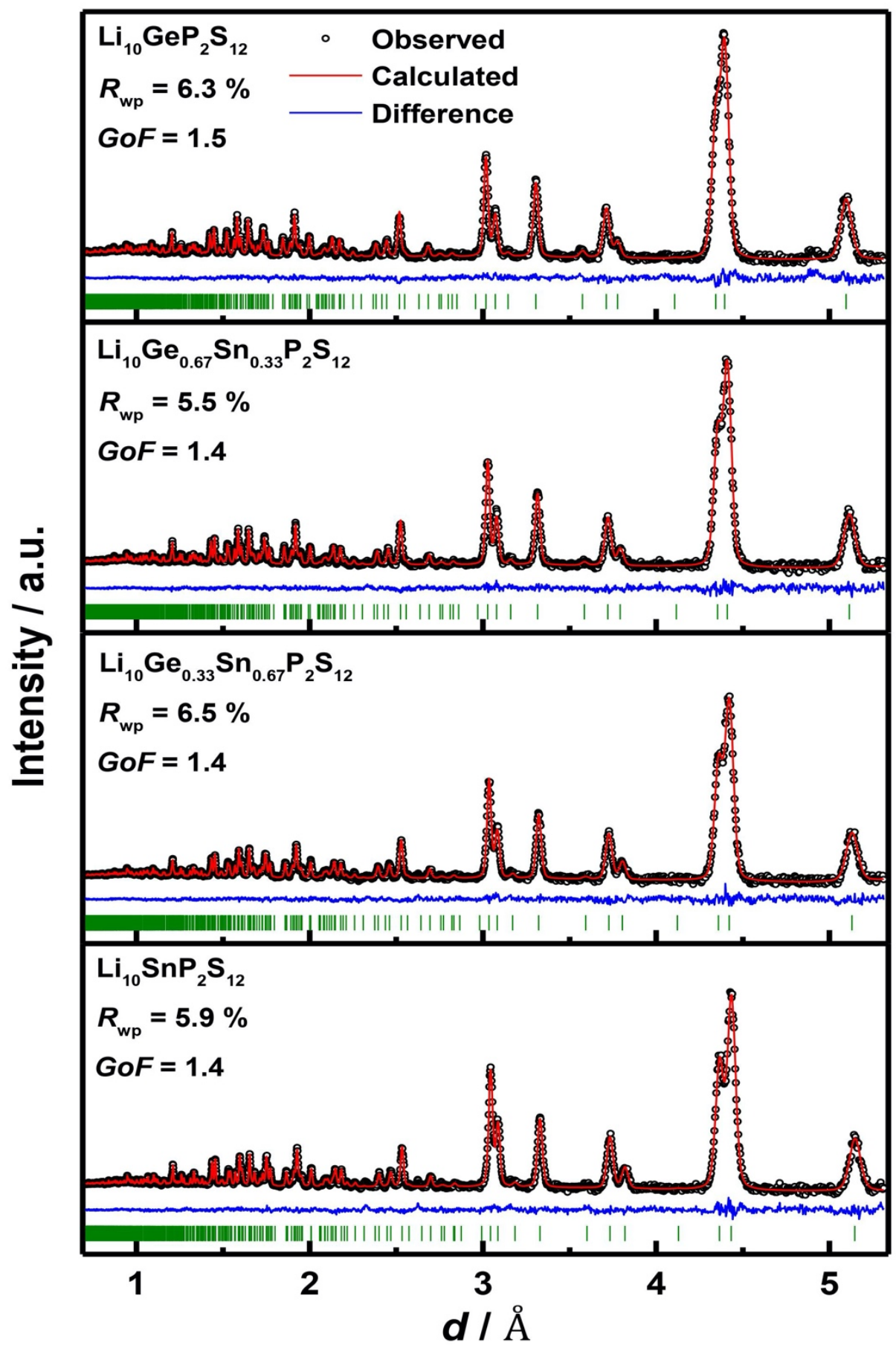

Figure S1: Representative Rietveld refinements of neutron diffraction data at $300 \mathrm{~K}$ for $\mathrm{Li}_{10} \mathrm{Ge} e_{\text {- }}$. ${ }_{x} S_{n} P_{x} P_{2} S_{12} . R_{w p}$ and GoF are the weighted profile residuals and goodness-of-fit, respectively. 
Table S1: Constraints used to refine the $\mathrm{Li}_{10} G e_{1-x} \mathrm{Sn}_{x} \mathrm{P}_{2} \mathrm{~S}_{12}$ structures from powder time-offlight neutron diffraction data.

\begin{tabular}{|c|c|c|c|c|c|c|}
\hline Atom & $\begin{array}{c}\text { Wyckoff } \\
\text { Site }\end{array}$ & $\mathrm{x} / a$ & $\mathrm{y} / b$ & $\mathrm{z} / c$ & Occ. & $\mathrm{B}_{\mathrm{eq}} / \AA^{2}$ \\
\hline Li1 & $16 h$ & Pos1 & Pos2 & Pos3 & {$[5-O c c 1-(4 *$ Occ 2$)-O c c 3] / 4$} & Var1 \\
\hline Li2 & $4 d$ & 0.25 & 0.25 & Pos4 & Occ1 & Var2 \\
\hline $\mathrm{Li} 3$ & $16 h$ & Pos5 & Pos6 & Pos7 & Occ2 & Var3 \\
\hline $\mathrm{Li} 4$ & $4 c$ & 0.25 & 0.75 & Pos8 & Occ3 & Var4 \\
\hline $\mathrm{Ge} / \mathrm{Sn} 1$ & $4 d$ & 0.25 & 0.25 & Pos9 & $0.5-\mathrm{Occ} 4$ & Var5 \\
\hline $\mathrm{Ge} / \mathrm{Sn} 2$ & $4 d$ & 0.25 & 0.25 & Pos9 & Occ4 & Var5 \\
\hline $\mathrm{P} 1$ & $4 d$ & 0.25 & 0.25 & Pos9 & 0.5 & Var5 \\
\hline P2 & $2 b$ & 0.25 & 0.75 & 0.75 & 1.0 & Var6 \\
\hline S1 & $8 g$ & 0.25 & Pos 10 & Pos11 & 1.0 & Var7 \\
\hline S2 & $8 g$ & 0.25 & Pos12 & Pos13 & 1.0 & Var8 \\
\hline S3 & $8 g$ & 0.25 & Pos14 & Pos15 & 1.0 & Var9 \\
\hline
\end{tabular}


a)

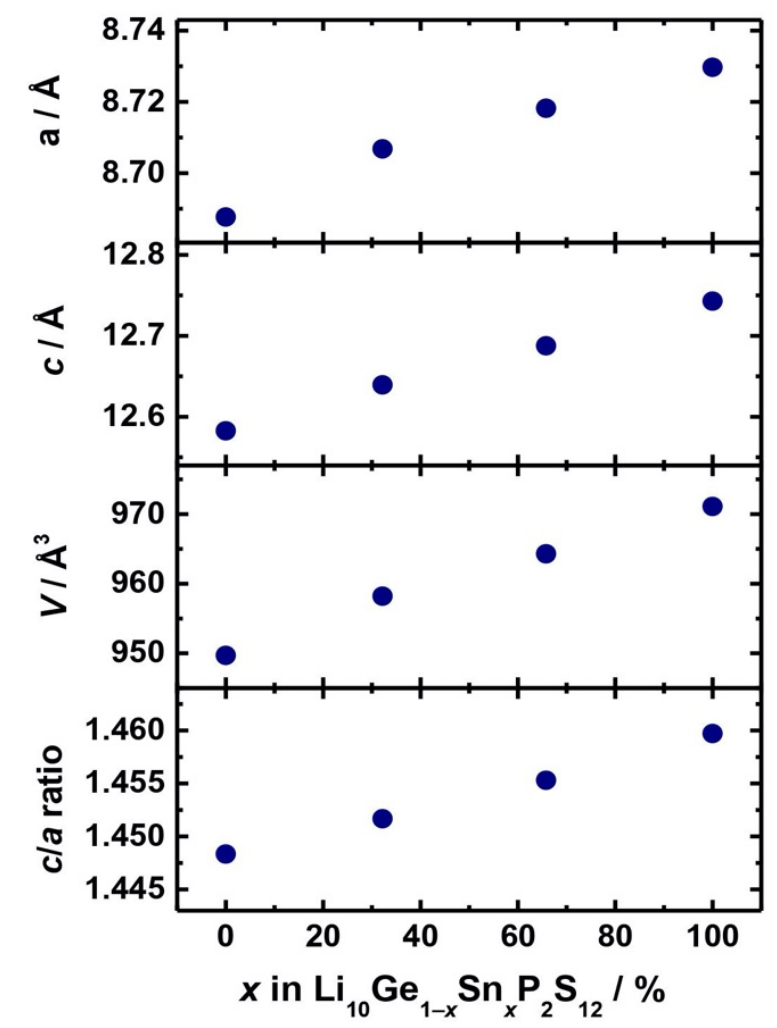

$\mathrm{Li}_{10} \mathrm{Ge}_{1-x} \mathrm{Sn}_{x} \mathrm{P}_{2} \mathrm{~S}_{12}$

b) $\bullet x=0 \bullet x=0.33 \bullet x=0.67 \bullet x=1$

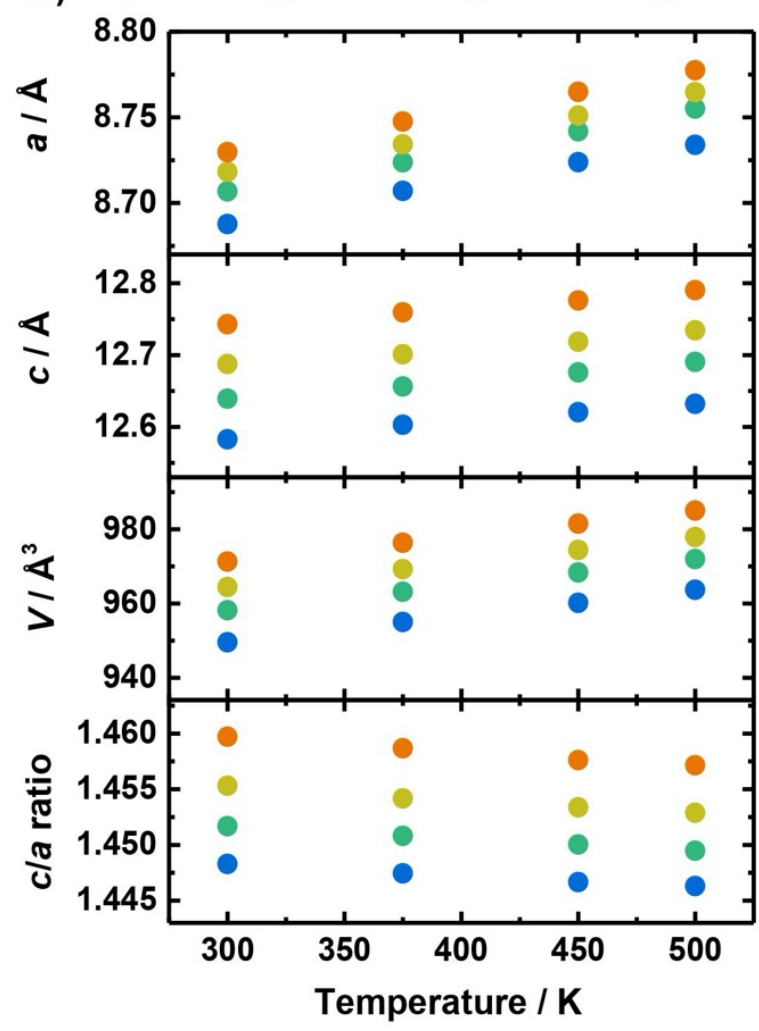

Figure S2: (a) Dependence of the room-temperature lattice parameters, lattice volume and c/a ratio in $\mathrm{Li}_{10} \mathrm{Ge}_{1-x} \mathrm{Sn}_{x} P_{2} \mathrm{~S}_{12}$ on the refined Sn occupancies. (b) Temperature dependence of the lattice parameters, lattice volume and cla ratio in $\mathrm{Li}_{10} \mathrm{Ge}_{1-x} \mathrm{Sn}_{x} \mathrm{P}_{2} \mathrm{~S}_{12}$.

a)

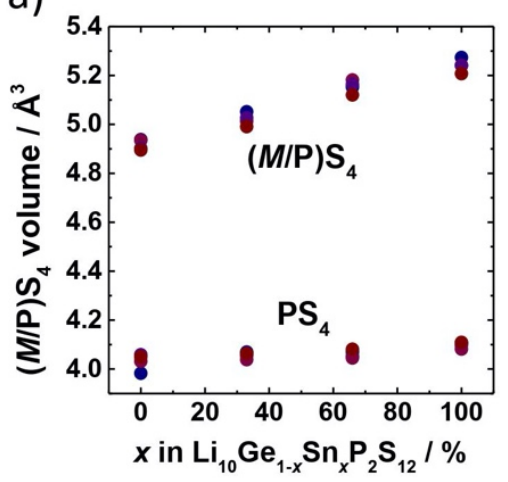

b)

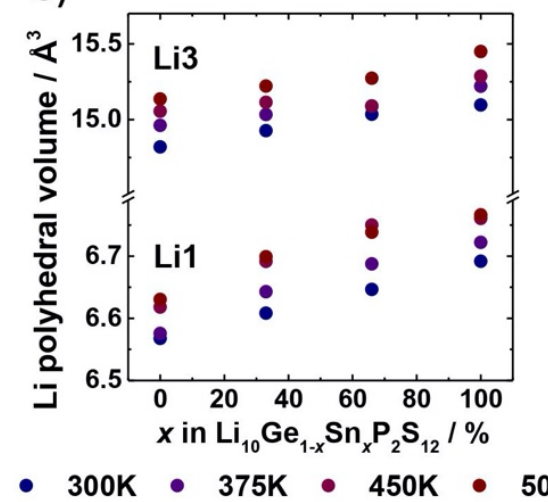

c)

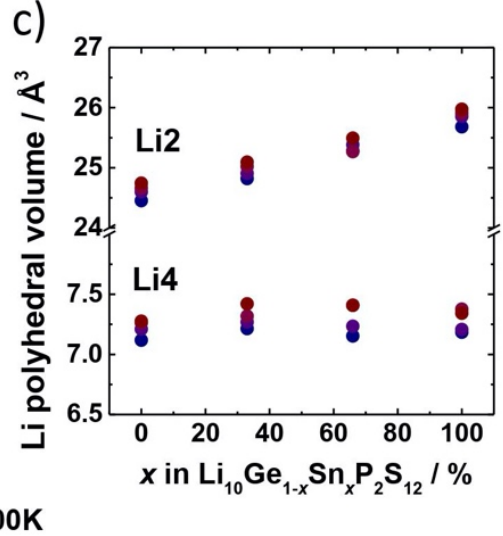

Figure S3: Effect of temperature and composition on (a) $P S_{4},(M / P) S_{4}$ and $(b, c)$ lithium polyhedral volumes. 

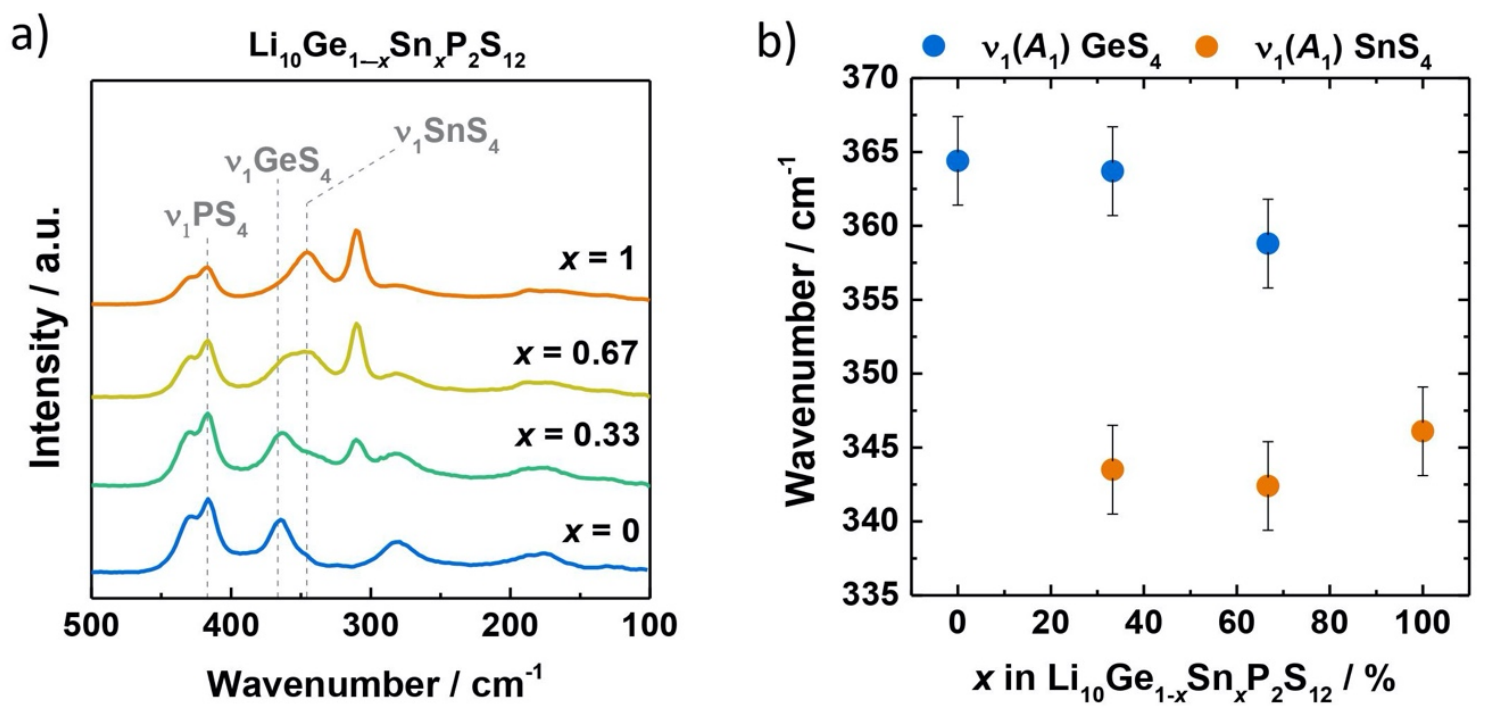

Figure S4: (a) Raman spectra with the relevant tetrahedral vibrations assigned. An additional Raman band at $310 \mathrm{~cm}^{-1}$ corresponds to 2-4 wt\% $\mathrm{Li}_{2} \mathrm{SnS}_{3}$ side phase. (b) Raman shifts for the symmetric stretching modes of the $\mathrm{GeS}_{4}$ and $\mathrm{SnS}_{4}$ tetrahedra. 

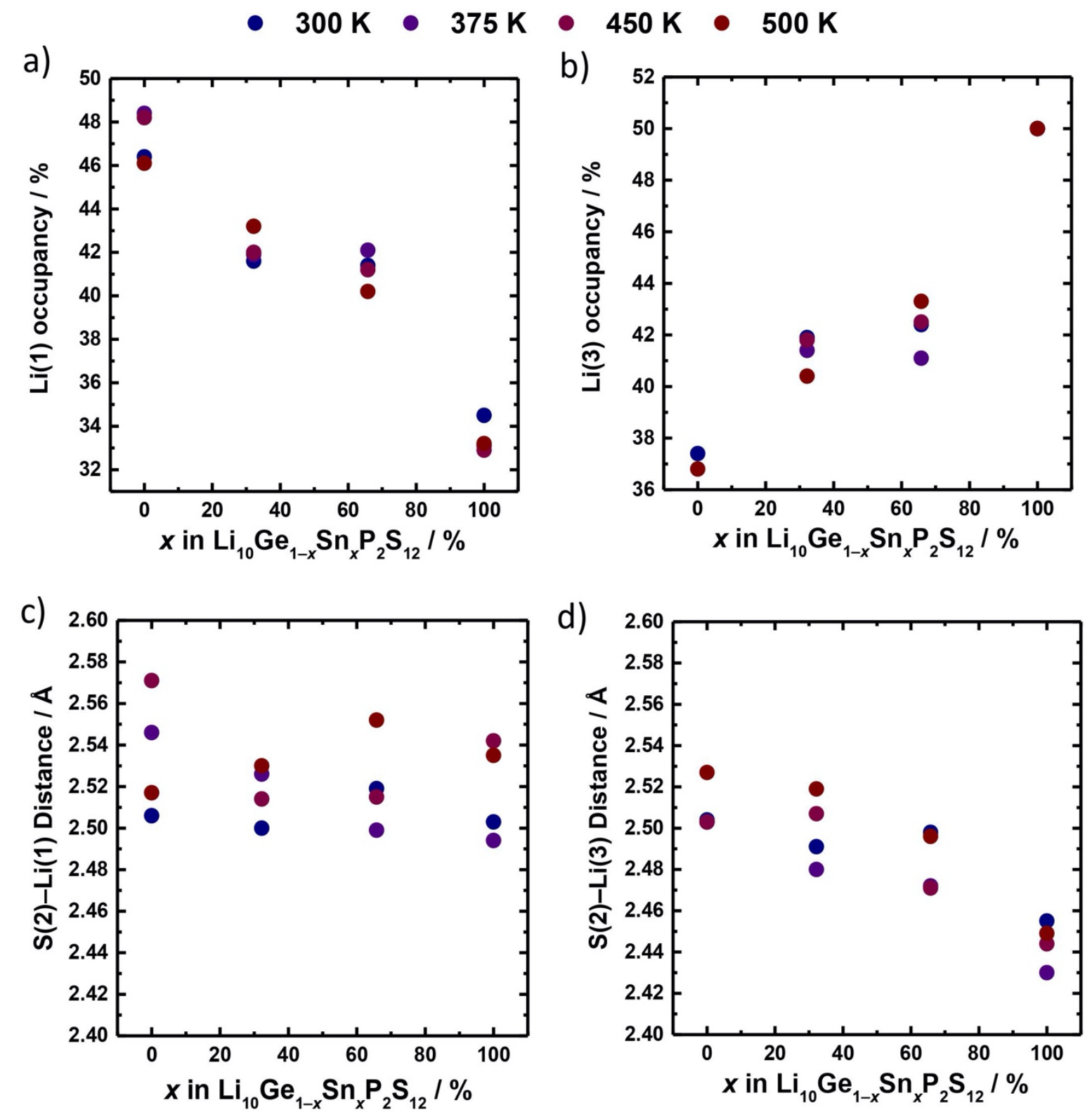

Figure S5: All data obtained from Rietveld refinements at each temperature relative to lithium occupancies $(a, b)$ and $S-$ Li distances $(c, d)$ 
Table S2: Room temperature ionic conductivity $\left(\sigma_{R T}\right)$ and activation energy $\left(E_{A}\right)$ of $L_{10} G e_{1-}$ ${ }_{x} S n_{x} P_{2} S_{12}$ from reference 35 .

\begin{tabular}{|c|c|c|}
\hline Composition & $\mathbf{E}_{\mathbf{A}} / \mathbf{e V}$ & $\boldsymbol{\sigma}_{\mathbf{R T}} / \mathbf{m S c m}^{\mathbf{- 1}}$ \\
\hline $\mathrm{Li}_{10} \mathrm{GeP}_{2} \mathrm{~S}_{12}$ & $0.274(3)$ & $7.6(3)$ \\
\hline $\mathrm{Li}_{10} \mathrm{Ge}_{0.66} \mathrm{Sn}_{0.33} \mathrm{P}_{2} \mathrm{~S}_{12}$ & $0.275(3)$ & $6.4(3)$ \\
\hline $\mathrm{Li}_{10} \mathrm{Ge}_{0.33} \mathrm{Sn}_{0.66} \mathrm{P}_{2} \mathrm{~S}_{12}$ & $0.285(3)$ & $4.8(2)$ \\
\hline $\mathrm{Li}_{10} \mathrm{SnP}_{2} \mathrm{~S}_{12}$ & $0.304(3)$ & $3.8(2)$ \\
\hline
\end{tabular}

